\title{
Call for Papers: Können wir Sie als Autor gewinnen?
}

Die Marketing Review St. Gallen verfolgt das Ziel, den Austausch zwischen Theorie und Praxis zu fördern. Alle Beiträge werden im Rahmen eines Double-Blind-Review-Prozesses anonym von einem Wissenschaftler und einer Marketingführungskraft begutachtet. Dadurch wollen wir die anwendungsorientierte Qualität der MRSG für den Leser sicherstellen. Beiträge können in deutscher oder englischer Sprache eingereicht werden.

Können wir auch Sie als Autor gewinnen?

\section{Inhaltliche Koordination der Ausgabe}

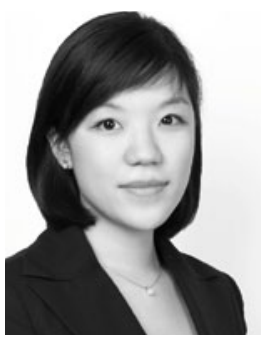

Dipl.-Kffr. You-Cheong Lee

Wissenschaftliche Mitarbeiterin am Institut für Marketing an der Universität St.Gallen (HSG).

\section{Grundsatzbeiträge}

In jeder Ausgabe werden einzelne Grundsatzbeiträge zu Marketingthemen aufgenommen. Voraussetzung ist, dass das Thema auf breites Interesse stößt und in sich geschlossen behandelt wird. Bitte senden Sie vorab einen Abstract (max. eine Seite) an sven.reinecke@unisg.ch und marketingreview@unisg.ch.

\section{Schwerpunktthemen}

Als Themenheft setzt die Marketing Review St. Gallen in jeder Ausgabe einen inhaltlichen Schwerpunkt.

Für das Jahr 2014 können leider keine Schwerpunktbeiträge mehr aufgenommen werden. Die Schwerpunktthemen für die Marketing Review St. Gallen 2015 sowie die entsprechenden Bewerbungsadressen erfahren Sie in Kürze online unter www.marketingreview.ch.

\section{Veranstaltungen}

\section{Veranstaltungen zum Thema Marketing}

\begin{tabular}{|c|c|c|}
\hline Datum & Event & Website \\
\hline $25.06-26.06 .2014$ & Mailingtage & www.mailingtage.de \\
\hline 26.08.-28.08.2014 & SuisseEMEX 2014 & www.suisse-emex.ch \\
\hline 30.08.-02.08.2014 & Webchance & www.webchance.de \\
\hline 10.09.-11.09.2014 & Dmexco & www.dmexco.de \\
\hline 08.10.-10.10.2014 & CRM-expo & www.where-it-works.de \\
\hline 01.10.-20.10.2014 & marke[ding]plus & www.markeding-plus.at \\
\hline 21.10.-22.10.2014 & ad:tech London & www.ad-tech.com \\
\hline 22.10.-23.10.2014 & $\begin{array}{l}\text { Research \& Results } \\
\text { München }\end{array}$ & www.research-results.com \\
\hline 22.10.-24.10.2014 & Medientage München & www.medientage.de/ \\
\hline 06.10.-07.10.2014 & Publisher's Summit 2014 & http://publishers-summit.vdz.de/ \\
\hline 13.11 .2014 & OWM-Fachtagung 2014 & www.owm.de \\
\hline
\end{tabular}

\begin{tabular}{|c|c|}
\hline Ort & Veranstalter \\
\hline Nürnberg & Nürnberg Messe \\
\hline Zürich & EMEX Management GmbH \\
\hline Frankfurt & $\begin{array}{l}\text { Messe Frankfurt Exhibition } \\
\text { GmbH }\end{array}$ \\
\hline Köln & Köln Messe, BVDW, OVK \\
\hline Stuttgart & Landesmesse Stuttgart GmbH \\
\hline $\begin{array}{l}\text { Wels, } \\
\text { Österreich }\end{array}$ & Messe Wels GmbH \& Co KG \\
\hline London & dmg :: events \\
\hline München & $\begin{array}{l}\text { Reitmeier Input Management } \\
\text { Services GmbH }\end{array}$ \\
\hline München & Medientage München GmbH \\
\hline Berlin & $\begin{array}{l}\text { Verband Deutscher Zeitschrif- } \\
\text { tenverleger }\end{array}$ \\
\hline Berlin & $\begin{array}{l}\text { Organisation Werbungtreibende } \\
\text { im Markenverband }\end{array}$ \\
\hline
\end{tabular}

